\title{
Modelling a New Product Model on the Basis of an Existing STEP Application Protocol
}

\author{
B.-R. Hoehn, K. Steingroever, M. Jaros
}

\begin{abstract}
During the last years a great range of computer aided tools has been generated to support the development process of various products. The goal of a continuous data flow, needed for high efficiency, requires powerful standards for the data exchange. At the FZG (Gear Research Centre) of the Technical University of Munich there was a need for a common gear data format for data exchange between gear calculation programs. The STEP standard ISO 10303 was developed for this type of purpose, but a suitable definition of gear data was still missing, even in the Application Protocol AP 214, developed for the design process in the automotive industry.

The creation of a new STEP Application Protocol or the extension of existing protocol would be a very time consumpting normative process. So a new method was introduced by FZG. Some very general definitions of an Application Protocol (here AP 214) were used to determine rules for an exact specification of the required kind of data. In this case a product model for gear units was defined based on elements of the AP 214. Therefore no change of the Application Protocol is necessary.

Meanwhile the product model for gear units has been published as a VDMA paper and successfully introduced for data exchange within the German gear industry associated with FVA (German Research Organisation for Gears and Transmissions). This method can also be adopted for other applications not yet sufficiently defined by STEP.
\end{abstract}

Keywords: data exchange, product model, gear data, STEP.

\section{Introduction}

The use of computers has become more and more common for the development of all kinds of products. CAD systems have been introduced successfully for the designing process. Additionally there are a lot of calculation and simulation tools to help the designer. However, keeping data digitized is not limited to the design process. Modern PLM systems (Product Lifecycle Management) are able to cover all data ocurring during the whole lifecycle of a product. Therefore powerful interfaces are required to handle the numerous different native data formats from different applications. The goal is an unlimited data flow from the start to the end of any product.

For the development process of gear units the gear calculation programs of FVA (Forschungsvereinigung Antriebstechnik e.V., German Research Association for Gears and Transmissions) [1] are very widely used tools. These programs were developed by several gear research institutes at the German universities. There is a great range among the programs from the calculation of the geometry, load capacity and efficiency of the different kind of gears to programs simulating shafts, bearings and housings. Nearly all aspects of a gear unit can be calculated and optimized. Although FVA has standards for the design of a new program, no data exchange among these programs was usually provided. This means even basic data like the number of teeth or the face width of a gear have to be transferred manually by the user. No common format for gear data existed for the use of all FVA programs. If some gear unit date is to be changed, the data has to be changed in the input files of all participating programs. This is an unwanted process with a high risk of errors.

These were the initial conditions some years ago that led to a project at FZG (Forschungsstelle fuer Zahnraeder und Getriebebau, Gear Research Centre of the Technical University of Munich) to improve the data exchange between the gear calculating programs of FVA. The main goal was to realize of a product model for gear units for use as a central data base. For the data exchange itself a converter program should be developed. With this standardized format only one interface per each program is needed. The powerful STEP standard ISO10303 [2] should be the basis for the product model for gear units. A second goal was to introduce data exchange between the gear calculation programs and CAD systems. Therefore the STEP standard is the only existing standard that could cover both kind of data.

Definitions for gear data do not exist sufficiently within the STEP standard. The typical way to define data in STEP as a so called Application Protocol would be a too time consumpting normative process. So the challenge was to create a new way to define gear data in STEP.

\section{Contents of the product model for gear units}

The first and main purpose for the product model for gear units is to realize the data exchange between the gear calculating programs of the German Research Association for Gears and Transmissions FVA. These programs are commonly used for the designing process of gears and gear units within the German gear industry. Therefore all data of machine elements occurring during the development process of a gear unit are covered by the product model.

Following elements are included in the product model for gear units:

- Gears: Spur and helical gears, bevel gears, hypoid gears, crossed helical gears and worm gears.

- Shafts

- Bearings: Plain bearings and rolling element bearings.

- Sealings

- Housings and basement 
- Clutches

- Brakes

- Couplings

Further elements not directly related to a gear unit are also included to the product model:

- Materials: Materials used for the above listed elements.

- Lubricants

- Tools: The tools are limited to tools used for gears.

All of these elements can be calculated by a wide range of FVA programs. The product model for gear units is to be used as a common format for central storage of all data belonging to these elements. The kind of data is very varied: Data related to geometry, load capacity, specification, life time, application, safety, efficiency, environment, manufacturing, etc. are contained in the product model. Not included are the electrical, electronic, hydraulic and pneumatic aspects of a gear unit.

\section{ISO 10303 STEP}

In order to develop a product model for gear units a suitable format is required. A very powerful format that includes not only geometrical data but also consists of rules for modelling any other product data is ISO 10303 STEP [2].

STEP means standard for the exchange of product data. It was developed to replace existing standards like IGES, DXF, VDA-FS and SET. The purpose of the older standards is to exchange geometric data between CAD systems. The capability of STEP also contains geometric data, but the power of STEP consists of the ability to include much more data. STEP covers manufacturing data, tolerances, qualities, material data, working schedules and cost structures. All data occurring during the life cycle of a product can be represented by STEP. STEP enables the creation of a modern, object oriented and system independent database. A wide range of data is already defined in STEP, and much more will be added in the near future.

\subsection{The structure of STEP}

ISO 10303 STEP is organized into different parts (see Fig. 1). Parts 1-14 are the parts that include the description methods. The EXPRESS description language is specified there. This is not a programming language, but a language specially generated to represent data. EXPRESS contains an object oriented structure with the basic element 'entity'. An entity describes a class of objects with the same attributes and properties. As in other object oriented languages, subtypes and supertypes are provided as well as the inheritance of attributes from the supertype to the subtype. For the visualization of EXPRESS the graphical representation EXPRESS-G is included in the STEP standard. EXPRESS-G represents the structure of the objects and attributes graphically within diagrams.

The basic parts are the integrated generic resources (parts 41-53). They contain the elementary definitions for geometry, topology, materials, tolerances, etc. Application related definitions are described in the integrated application resources (parts 101-110). These are definitions for draughtings, kinematics, finite element analysis, etc.

The application protocols are defined for special applications. They contain the determination of the boundary conditions, the scope of the application protocol, and the usage and interpretation of the elements of the integrated generic resources and the integrated application resources required for that application. The application protocols are assigned to a specific industrial sector. The application protocol AP 214 [3] for the automotive industry is one of the application protocols with a publication stage. About 40 application protocols are already in existence or are under development.

\begin{tabular}{|c|c|}
\hline \multicolumn{2}{|l|}{ ISO 10303} \\
\hline $\begin{array}{l}\text { Part } 1 \text { - 14: } \\
\text { DESCRIPTION METHODS } \\
\text { (e.g. EXPRESS language) }\end{array}$ & $\cdots$ \\
\hline $\begin{array}{l}\text { Parts } 41 \text { - 53: } \\
\text { INTEGRATED GENERIC RESOURCES } \\
\text { (Geometry, Topology, Materials, Tolerances, } \\
\text { Representation,...) }\end{array}$ & $\begin{array}{l}\text { Parts } 101-110 \text { : } \\
\text { INTEGRATED APPLICATION RESOURCES } \\
\text { (Draughting, Kinematics, Finite element } \\
\text { analysis, ...) }\end{array}$ \\
\hline \multicolumn{2}{|l|}{$\cdots$} \\
\hline \multicolumn{2}{|c|}{$\begin{array}{l}\text { Parts } 201-240 \text { : } \\
\text { APPLICATION PROTOCOLS } \\
\text { - ... } \\
\text { - AP } 212 \text { Electronical design and installation } \\
\text { - AP } 214 \text { Core data for automotive mechanical design process } \\
\text { - AP } 226 \text { Ship mechanical systems } \\
\text { - ... }\end{array}$} \\
\hline$\cdots$ & \\
\hline
\end{tabular}

Fig. 1: Structure of STEP [2] 
Other parts of STEP are the implementation methods (parts 21-29), the conformance testing methods and framework (parts 31-35) and the application interpreted constructs (parts $50-522$ ).

\subsection{Gear data inside ISO 10303 STEP}

The only gear data in the scope of ISO 10303 STEP is included in the application protocol AP 214. There, the entity GEAR_PAIR is defined. This GEAR_PAIR is a subtype of a KINEMATIC_PAIR and owns five attributes (see Fig. 2): two radii, one bevel angle, one helical angle, and the gear ratio.

These properties are of course not sufficient for a detailed description of a whole gear unit itself. They only give information about the mechanical coupling of two objects. No more data about the gear unit is provided inside the scope of the application protocol AP 214 of STEP.

The idea to add more attributes to the GEAR_PAIR is not in correspondence with the application protocol AP 214. In this case any conformity with this existing standard would be lost. An extension of the application protocol AP 214, which was originally generated to describe the design process in the automotive industry, with more gear data would result in a very time consuming normative process.

Gear unit data is not limited to gears and gear pairs. Shafts, bearings, sealings and couplings also belong to a complete gear unit. Therefore an extension based on the GEAR_PAIR is not a sufficient solution.

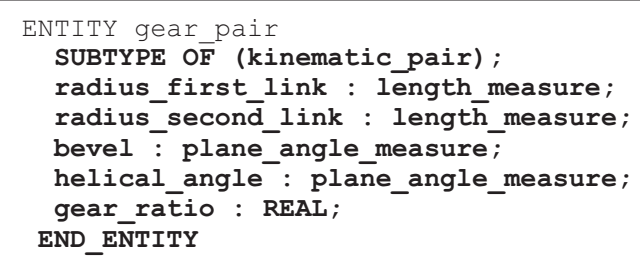

Fig. 2: Entity GEAR_PAIR

\subsection{Methods to create a new STEP product model}

There are two ways to generate a new product model in STEP (see Fig. 3). The first possibility would be to create a new application protocol similar to AP 214 for the automotive industry. A complete definition specially for gear data can be created in this way. The problem is that the realization of a new application protocol means a very laborious and time-consuming procedure taking years to get completed.

The second way is to extend an existing application protocol. For the product model for gear units the application protocol for the automotive industry is the most suitable one, and already includes an entity GEAR_PAIR (see chapter 3.2). Other objects (e.g. shafts, bearings) of a gear unit are difficult to integrate into this GEAR_PAIR, so some new entities (and the surrounding structure) have to be created. A change in the structure of this application protocol would cause loss of compatibility with the existing standard and a difficult normative process would have to be undertaken to integrate the new extensions.

\section{Integration of the product model for gear units into ISO 10303 STEP}

For the demands of the product model for gear units a new method had to be developed by the Gear Research Centre (FZG) of the Technical University of Munich. This method uses the structure of the existing AP 214 but is completely independent. A new mechanism ensures total compatibility with AP 214 although new objects are included inside this model. This method does not need the complicated normative process of a new application protocol.

\subsection{Gear data definitions using existing AP 214 definitions}

AP 214 contains many general definitions that can be specified more exactly be the user himself. E.g. there is the entity (or object) called an 'item': "An item is either a single object or a unit in a group of objects. It collects the information that is common to all versions of the object." ([3], chapter 4.2.267). This 'item' can be used for any purpose. The entity 'Specific_item _classification' is linked to that 'item' and provides some classification attributes. "A Specific_item_classification is a classification of an item with respect to specific criteria. The specific criteria are covered in the 'classification_name' attribute." ([3], chapter 4.2.465). There are several predefined values for the 'classification_name' within AP 214. These are 'part', 'prototype', 'assembly', 'collection', 'detail', 'raw material', etc. If applicable the predefined values shall be used. In the case of requiring some definitions not included in the proposed values, it is allowed to introduce additional definitions. This means that it is possible to define gear data within the scope of the already existing AP 214 by oneself.

For the application as gear data the 'classification_name' of the entity 'specific_item_classification' can be set to names like 'gear', 'helical gear', 'spur gear', 'bevel gear', 'shaft', 'bearing', 'sealing', 'housing', etc. This way the classified entity 'item' is clearly defined as an element of a gear unit (see Fig. 4).

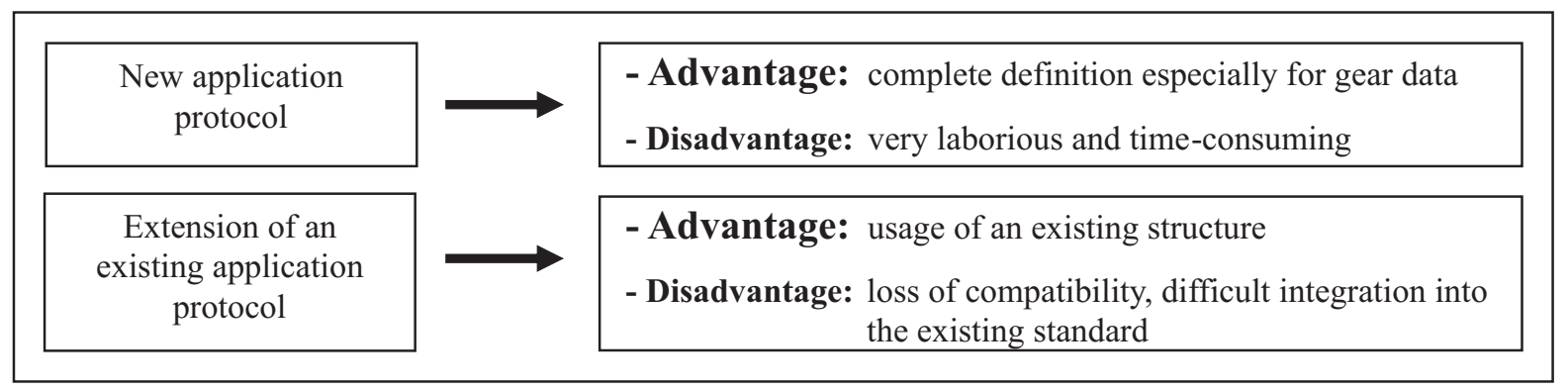

Fig. 3: Methods for creating a product model 


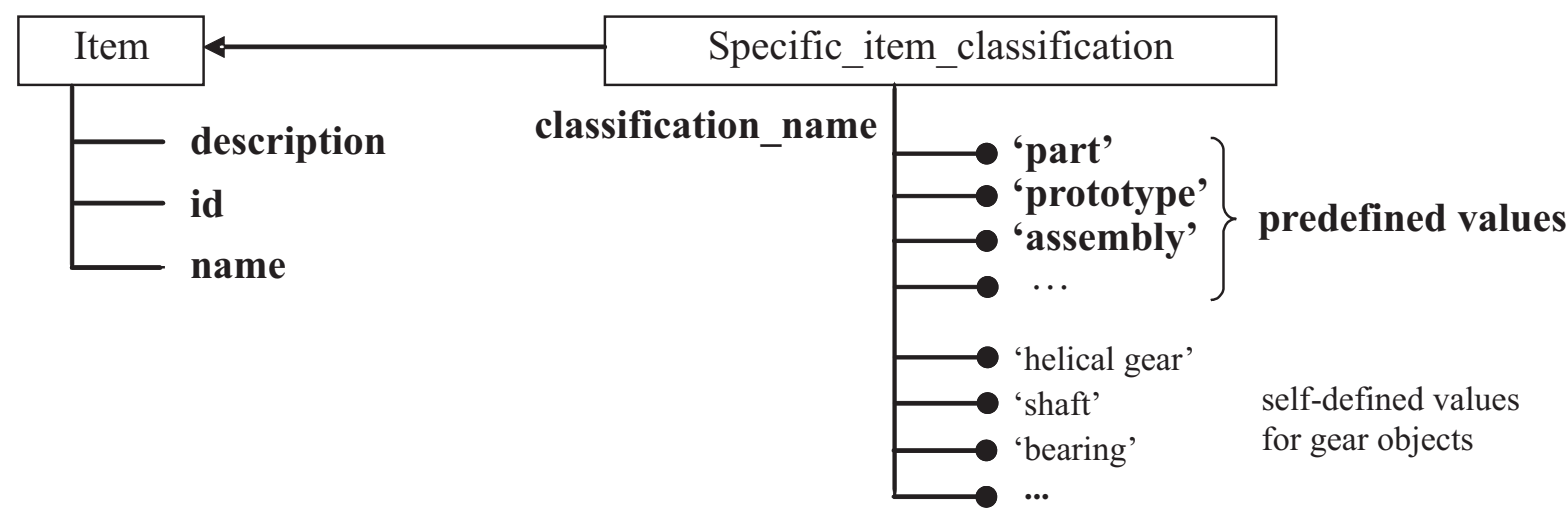

Fig. 4: Self-defined classification_name

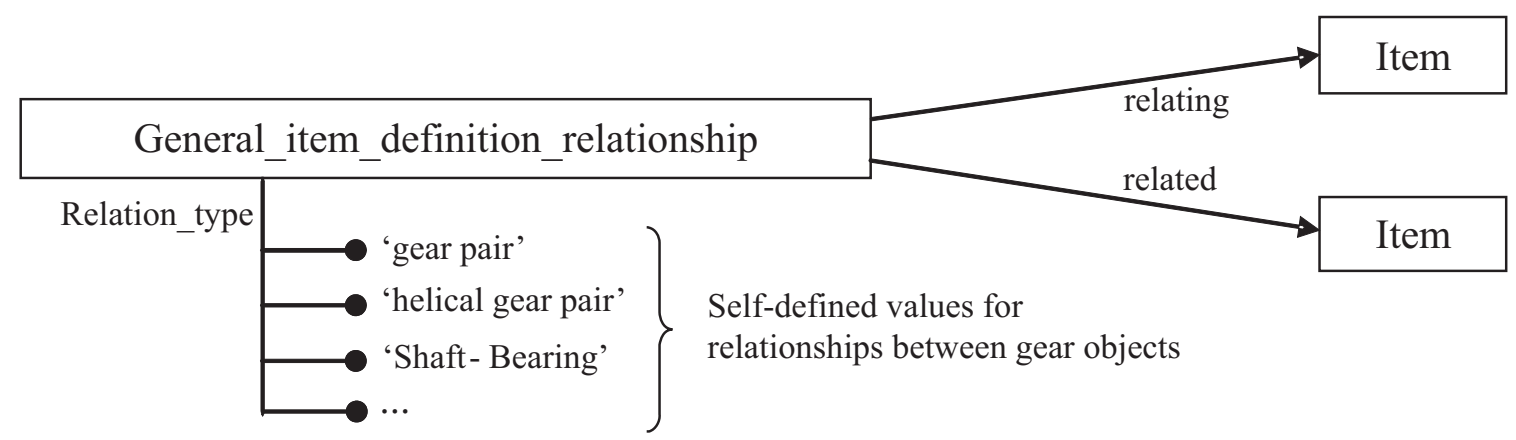

Fig. 5: Self-defined relationships

All required elements of a gear unit can be covered by the entity 'item' using self-defined names. To determine the relations between two gear elements, e.g. the linking of two gears within a gear pair, additional objects from AP 214 are needed (see Fig. 5). The entity 'General item definition relationhip' defines the relation between exactly two 'items'. It contains an attribute 'relation_type' that can also be used for self-defined values. This way the relationships between the gear elements can be introduced. These are the different kinds of gear pairs like spur gear pair, helical gear pair, bevel gear pair and worm gearing, and also relations between two shafts, two bearings or between shafts and bearings, etc. Assemblies, such as the complete gear unit or a planetary gear unit inside the gear unit can aslo be defined by the 'item' and the 'specific_item_classification'. The predefined values contain an 'assembly', which can also be used for gear units. For a specific assembly of a gear unit a second 'specific_item_classification' with the self-defined name is to be added.

Fig. 6 shows an example of an assembly 'planetary gear unit' with the gears and the relationships between the gears

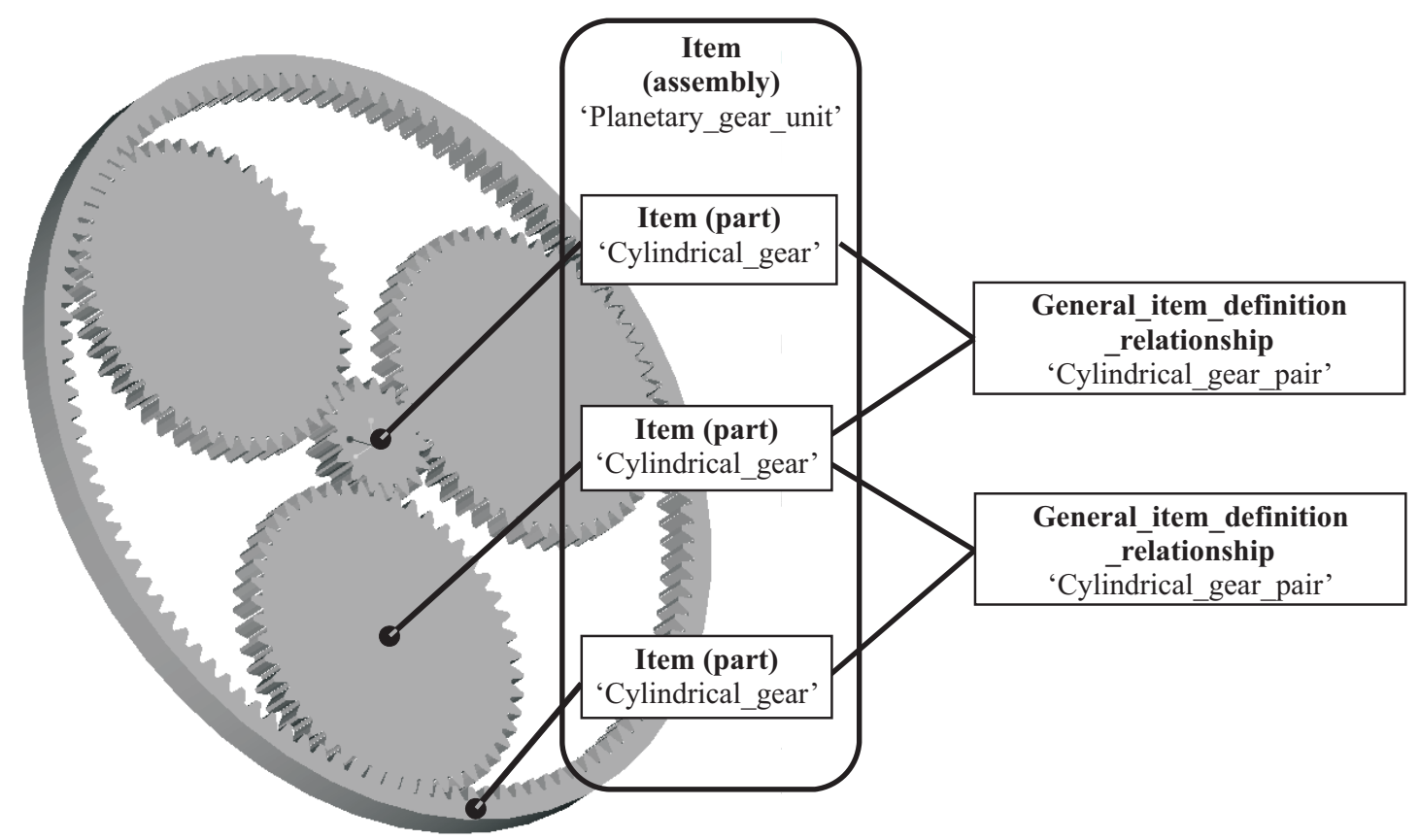

Fig. 6: Planetary gear unit: usage of AP 214 objects with self defined attributes 


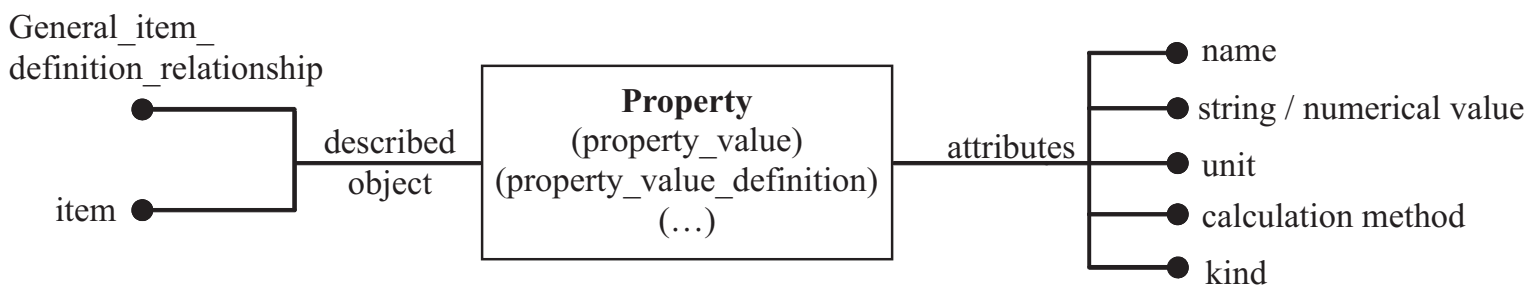

Fig. 7: Data covered by property

using self-defined values for the AP 214 objects. An 'item' can belong to several relationships and assemblies.

For the definition of the data of these objects STEP AP 214 provides a structure called a 'property' (see Fig. 7). This 'property' consists of several entities that indicate information belonging to an object. For the application to gear data the 'property' can indicate the value (string value or numerical value) itself, the unit of the value, the used calculation method for determining the value and the kind of the data (geometry, load capacity, life time, safety, etc.). This property is linked to the relating object. This can be an 'item' as well as a 'general_item_definition_relationship'. In principle all data belonging to the gear elements can be covered by the 'property'.

The figures show simplified extracts of the AP 214 structure. The complete structure includes some more elements and relations not shown in the diagrams.

\section{Problems:}

Why cannot STEP AP 214 be used for gear data in the way shown above? The main problem is that it is only one way to define data by oneself. There are also other elements within AP 214 that are suitable for determining gear data. Even usage of the proposed objects does not mean a standardized solution. Different names could be set for the self-defined values. E.g. the classification name indicating a gear can be called 'gear', 'cylindrical gear', 'helical gear' or 'spur gear'. The are no standardized names determined within AP 214, and even foreign language names can be set. This problem occurs not only for the objects but also for the property names. If not clearly defined, the data cannot be recognized by any electronic program. The complex structure of STEP requires electronic data processing. For the inter- pretation of gear data in STEP detailed explanations have to be added in this case.

The definition of gear data using self-defined values can only work internally within a company. This method is not suitable for general application because there is no clear standardization.

\subsection{Standardized specification for the product model for gear units}

The main problem in using STEP AP 214 for gear data is the lack of any standardization. Therefore the objects and structures needed from AP 214 and the names of the gear data have to be determined in a standardized way.

Therefore the product model for gear units developed by FZG contains clear definitions how to use AP 214 for defining gear data. All required elements of AP 214 are described exactly. The names outside the scope of AP 214, which have to be set for the gear data, are predefined in the product model for gear units.

For spur and helical gears the common name cylindrical gear was introduced. The definition of a cylindrical gear object gives clear information about the application of objects of AP 214. Translation tables for each object defined in the product model for gear units give complete information about the AP 214 elements and the relations that are required for the gear data object. Table 1 shows the translation table for the object 'cylindrical gear'. There are two links to the AP 214 object 'Specific item_classification', the first time using the self-defined value 'cylindrical_gear', the second time set with the predefined classification name 'part'. The principle of the table was adopted from the AP 214 mapping tables. There the

Table 1: Translation table for 'cylindrical_gear'

\begin{tabular}{|c|c|}
\hline Cylindrical_gear & Item \\
$\{[$ Item $<-$ \\
Specific_item_classification.associated_item \\
Specific_item_classification \\
Specific_item_classification.- \\
classification_name='cylindrical gear'] \\
{$[$ Item<- } \\
Specific_item_classification.associated_item \\
Specific_item_classification \\
Specific_item_classification.- \\
classification_name='part']\} \\
\hline
\end{tabular}


Table 2: Gear objects defined within the product model

\begin{tabular}{|l|l|l|l|}
\hline Parts & Relationships & Property kind & Property name \\
\hline Cylindrical_gear & Cylindrical_gear_pair & Geometry & number of teeth \\
Bevel_and_hypoid_gear & Bevel_and_hypoid_gear_pair & Load capacity & width \\
Shaft & Shaft_shaft & Safety & outer diameter \\
Plain_bearing & Shaft_bearing & Temperature & inner diameter \\
Rolling_element_bearing & Bearing_bearing & Torque_force & safety factor \\
Sealing & & $\ldots$ & application factor \\
$\ldots$ & & & $\ldots$ \\
\hline
\end{tabular}

same mechanism is used to match the objects of the application protocol to the basic STEP objects.

The product model for gear units introduces some different types of gear objects by using this mechanism. The main objects are shown in Table 2.

The property names are not related to a specific object. If applicable they can be used for any object. 'Number of teeth' is usually designated for gears, while 'width' can be generally used for gears, bearings or sealings. There are some more elements existing within the product model for gear units:

- Shape: This is a defined part of a 'part'. Maybe the section of a shaft or a tooth of a gear. Properties can also be assigned to a shape.

- List: The properties can be organized within lists. A list is a special kind of property that contains a list of other properties.

- Position_orientation_property: Some properties need information about the position or direction. E.g. values for forces or shifts can contain different values depending on their position or direction. For a clear distinction a position_orientation_property can be linked to any property.

\subsection{Publication and application of the product model for gear units}

The product model for gear units is published by the German VDMA (Verband deutscher Maschinen- und Anlagenbau e.V.) as a VDMA-paper [4] in English language. By using this VDMA-paper a calculation program related to gears can be adapted to the STEP product model for gear units by using a converter program which allows data transfer between the STEP format and the native format.

The product model for gear units was adapted to some programs of the German Research Association for Gears and Transmissions FVA (Forschungsvereinigung Antriebstechnik e.V.). In orders to realize a data exchange between the gear calculation programs a converter program was developed. This converter program can transfer data from and to the FVA programs. All data is stored in a common data base according to the STEP product model for gear units (see Fig. 8).

Data exchange to the FVA programs STplus [5] (calculation of geometry and load capacity of spur and helical gears) and RIKOR [6] (calculation of tooth modifications) is already successfully in service. The connection to 5 more FVA programs is now in process.

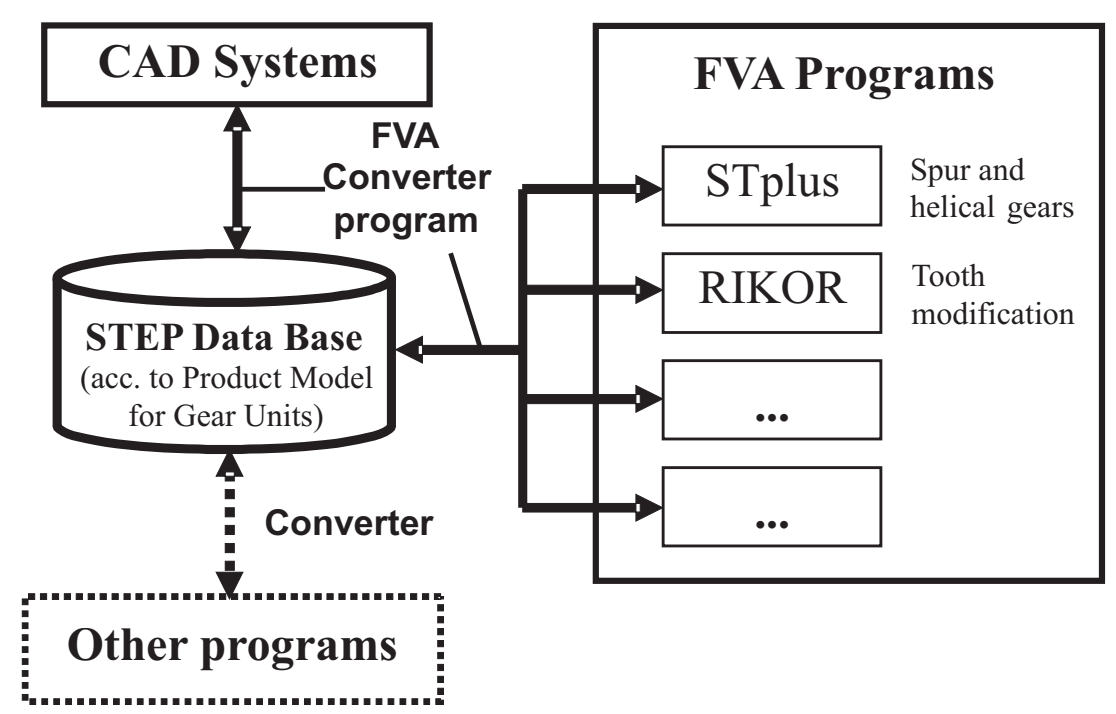

Fig. 8: Applications of the STEP product model for gear units to FVA programs 
The FVA converter program can also handle data exchange with CAD systems. A CAD system equipped with a STEP interface can read geometric data but is not able to interpret gear data according to the STEP product model. So the converter program transforms the gear data into geometric data. The shafts, gears and bearings become simple solids with defined shells, edges and points according to the geometrical definitions of AP 214. Data can be exchanged in both directions.

\section{Conclusion}

For data exchange between gear calculation programs a product model for gear units was developed at the Gear Research Centre FZG of the Technical University of Munich. This product model is based on application protocol AP 214 of ISO 10303 STEP. A new method defines the gear data as self-defined values of AP 214. This ensures $100 \%$ compatibility with the existing application protocol that was originally generated for the mechanical design process in the automotive industry. The rules for the use of AP 214 elements and the self-defined values are determined within the product model to get a standardized format.

The product model for gear units covers all data of a gear unit. These are not only gear data but also data related to shafts, bearings, lubricants and other mechanical gear elements. The product model for gear units is published by the German organisation VDMA as a paper in English language.

The first application successfully using the product model for gear units is the German Research Association for Gears and Transmissions FVA. A converter program was developed to realize data transfer from the FVA gear calculation programs to a central STEP data base for gear units. This converter program also enables data exchange with CAD systems via STEP. With the product model for gear units and the converter program the management of gear data is improved significantly. The data exchange of gear data between different parties is also simplified by this common format. Other programs related to gears can be adapted to this product model in the future.

\section{References}

[1] FVA-Merkblatt 0/11: Programmierrichtlinie für FORTRAN - Programme der FVA. FVA, 2000.

[2] ISO-Norm 10303-1: Industrielle Automatisierungssysteme und Integration - Produktdatendarstellung und -austausch Teil 1: Überblick und grundlegende Prinzipien. Berlin: Beuth, 1994.

[3] ISO-Norm 10303-214: Industrielle Automatisierungssysteme und Integration - Produktdatendarstellung und -austausch - Teil 214: Anwendungsprotokoll: Datenmodelle für die Prozesskette Mechanik in der Automobilindustrie. Berlin: Beuth, 2001.

[4] VDMA-Paper 23900: Recommendation for a Product Model for Gear Units. Beuth-Verlag, 2003.

[5] Steingroever, K.: FVA-Stirnradprogramm STplus, Benutzeranleitung Version 4. FVA-Heft 477, 2003.

[6] Schinagl, S.: FVA-Ritzelkorrekturprogramm RIKORG, Benutzeranleitung Version 1.0; FVA-Heft 481, 1999.

Prof. Dr. -Ing. Bernd-Robert Hoehn

phone: 0049 (0)89 28915807

e-mail: fzg@fzg.mw.tum.de

Dr. -Ing. Karl Steingroever

Dipl. -Ing. Michael Jaros

Gear Research Centre (FZG)

Internet: www.fzg.mw.tum.de

Technical University of Munich

Boltzmannstr. 15

85784 Garching

Germany 